\title{
Lecithin Based Pseudoternary Phase Diagrams
}

\author{
M. GosenCA, M. GAŠPERLIN
}

Faculty of pharmacy, University of Ljubljana, Ljubljana, Slovenia

E-mail: mirjam.gosenca@ffa.uni-lj.si (M. Gosenca)

Sci Pharm. 2010; 78: 690

doi:10.3797/scipharm.cespt.8.PPAT01

Aim: Different promising colloidal delivery systems including microemulsions and liquid crystals could be obtained with suitable combination of oil, surfactants/cosurfactants and water. However, the main problem from a formulation point of view has been to choose the surfactants capable of forming such systems and be physiologically acceptable in the same time. To overcome the later shortcoming the use of less toxic non ionic or biocompatible naturally obtained surfactant is proposed [1].

The aim of this study was to develop and characterize colloidal systems for dermal delivery based on nontoxic components, i. e. lecithin and Tween 80 as surfactant mixture, isopropyl myristate as oily phase and water.

Experimental: Pseudoternary phase diagrams were constructed using conventional titration technique with 1:1 and 2:1 mass ratios of Tween 80 to lecithin, respectively, isopropyl myristate and water. Clear samples of high viscosity were deemed to be lyotropic liquid crystals. Representative samples were further evaluated for their physical stability and rheological characteristics, while the structure of the samples was examined with a polarization microscope.

Results and discussion: The region of clear and highly viscous mixtures was detected at higher concentrations of Tween 80/lecithin (at both mass ratios) that were further on confirmed as lamellar lyotropic liquid crystals with polarization microscopy due to characteristic "Maltese cross" textures The samples were physically stable at room temperature during 2 months and after centrifuge test. Determination of viscosity and flow curves showed shear-thinning characteristics and thixotropic behaviour of samples containing more than $35 \%$ of water, while samples with less than $30 \%$ of water revealed rheopectic properties. Additionally, the viscosity was decreasing with increasing temperature, and was affected by composition of surfactant mixture (higher content of Tween 80 induced higher viscosity) and water content (up to $40 \%$ water content increased viscosity due to hydration of lecithin polar head groups).

Conclusion: Lamellar lyotropic liquid systems were identified from phase diagrams at higher surfactant concentration. They show great potential for dermal application due to nontoxicity of surfactants, physical stability and moreover, convenient rheological properties.

[1] Moreno MA, Ballesteros MP, Frutos P. Lecithin-Based Oil-inWater Microemulsions for Parenteral Use: Pseudoternary Phase Diagrams, Characterization and Toxicity Studies. J Pharm Sci. 2003; 7: 14281437. doi:10.1002/jps.10412 